\title{
THREE DIMENSIONAL RECONSTRUCTION WORKFLOWS FOR LOST CULTURAL HERITAGE MONUMENTS EXPLOITING PUBLIC DOMAIN AND PROFESSIONAL PHOTOGRAMMETRIC IMAGERY
}

\author{
W. Wahbeh ${ }^{\mathrm{a}, *}$, S. Nebiker ${ }^{\mathrm{a}}$ \\ ${ }^{a}$ FHNW, Institute of Geomatics Engineering, 4132 Muttenz, Switzerland - (wissam.wahbeh, stephan.nebiker)@fhnw.ch
}

Commission II

KEY WORDS: Photogrammetry, Reconstruction, Heritage, Monuments

\begin{abstract}
:
In our paper, we document experiments and results of image-based 3d reconstructions of famous heritage monuments which were recently damaged or completely destroyed by the so-called Islamic state in Syria and Iraq. The specific focus of our research is on the combined use of professional photogrammetric imagery and of publicly available imagery from the web for optimally 3d reconstructing those monuments. The investigated photogrammetric reconstruction techniques include automated bundle adjustment and dense multiview 3d reconstruction using public domain and professional imagery on the one hand and an interactive polygonal modelling based on projected panoramas on the other. Our investigations show that the combination of these two image-based modelling techniques delivers better results in terms of model completeness, level of detail and appearance.
\end{abstract}

\section{INTRODUCTION}

Photogrammetry has long been playing an important role in the documentation of cultural heritage monuments and sites. In situations where monuments are either destroyed or inaccessible, it offers the only possible solution for a digital reconstruction and/or documentation. The new political and social situation in the Middle East generated an enemy of cultural heritage who systematically destroys heritage monuments in the region. Many of them are amongst the oldest heritage monuments in the world and are listed as UNESCO world heritage sites. In the research for the virtual reconstruction of damaged and destroyed monuments in war zones especially in Syria, where we don't already have high accuracy surveys, it is clear that we cannot use other methods than photogrammetry in order to accurately reconstruct virtually the previous state of the monuments. Many recent professional and amateur projects are treating the topic based on huge amount of crowd-sourced touristic photos available on the web under open-domain copyright. For example, the platform "rekrei.com" are collecting volunteer's images of heritage monuments and making them available to anyone interested in heritage, which can help sorting and reconstructing. In addition, projects such as "Curious Travellers" or visualisingheritage.org are platforms for receiving photographs and videos from the public in order to be used in $3 d$ reconstructions by the project team. However, to our knowledge there are very few scientific publications providing background information or quality assessments of the reconstructions from crowd-sourced images.

Earlier attempts of such image-based 3d reconstructions had been triggered by the destruction of the Great Buddha statues of Bamiyan, Afghanistan by the Taliban in 2001. Gruen et al. (2002) had carried out a photogrammetric 3d reconstruction using only three metric images acquired in 1970. Nowadays, finding and collecting internet images for a prominent specific structure is quite easy. Images can be searched and classified on platforms such as "Flickr" or Google - based either on georeferencing tags included automatically in the images or on description tags added by the publishers. In cases of prominent monuments, such as those presented below, not only crowd-sourced public domain imagery but also professional metric or semi-metric imagery might be available. Combining these two image sources is expected to lead to significantly better 3d reconstruction results, than those achievable with just one of these data sets.

The goal of this paper is to study the strengths and limitations of photogrammetric reconstructions based on professional panoramic imagery on the one hand and on open domain touristic imagery on the other. Our main contribution are novel photogrammetric workflows combining these two image types in order to obtain 3d models that provide an optimum level of detail, accuracy, and completeness. The presented studies were carried out using data from four important but destroyed Syrian monuments. The studies are part of our overall research goal of permitting efficient and accurate $3 \mathrm{~d}$ reconstructions of destroyed and/or no longer accessible cultural heritage monuments.

Following a short summary of related work in section 2, we introduce the four monuments, their characteristics and the available data sets in section 3 . We dedicate the subsequent sections 4-6 to three different photogrammetric reconstruction approaches: panoramic image-based modelling, dense multiview reconstruction, and the new hybrid reconstruction process.

\section{RELATED WORKS}

The quality, type, quantity and distribution of the available imagery strongly influence the photogrammetric reconstruction techniques and their results. Depending on the technique used, we can expect differences in modelling approach, input imagery requirements and workflows, which might lead to significantly different results. These include different mesh topologies of the $3 \mathrm{~d}$ models, accuracies and representation outputs. Subsequently, we will look into related work either dealing with metric and semi-metric 'professional' imagery or with non-metric 'crowdcontributed' imagery.

3d reconstructions from metric and semi-metric imagery typically captured by professionals - are a well-researched topic in photogrammetry. Recent works, relevant to our research, 
investigate the application of Structure-From-Motion (SfM) and Dense Multi-View 3D Reconstruction (DMVR) algorithms to imagery acquired with DSLR cameras and the resulting accuracies Koutsoudis et al. (2014). Other authors such as Kersten and Lindstaedt (2012) or Santagati et al. (2013) compare the $3 \mathrm{~d}$ reconstruction results from metric imagery with results from terrestrial laserscanning. Alsadik et al. (2014) further discuss the important aspects of image network geometry for $3 \mathrm{~d}$ modelling in cultural heritage. While the above-mentioned works mainly focus on 'standard' imagery from pin-hole cameras, there are also a number of related investigations of exploiting panoramic imagery for the 3d reconstruction of cultural heritage monuments (Fangi, 2007; Fangi and Wahbeh, 2013). These professional photogrammetric reconstructions with wellcontrolled sensors and image networks stand in contrast to numerous research efforts exploiting the potential of unordered web-based image collections for efficiently reconstructing largescale urban environments (e.g. Agarwal et al., 2011). Much of this research places its focus on feasibility, performance and visual appearance. More recent work, e.g. by Wahbeh and Nebiker (2016), also quantitatively assessed the quality of the resulting $3 \mathrm{~d}$ reconstructions. The authors also investigated image acquisition patterns by tourists for typical heritage monuments following earlier investigations at urban scales by Kádár \& Gede (2013).

Wahbeh and Nebiker (2016) identified some typical problems with open domain tourist images and with subsequent $3 d$ reconstructions. These include: the presence of numerous uncalibrated cameras; large differences in image quality, resolution and lighting conditions; and very unevenly distributed image coverage. Typically, tourists are more interested in prominent parts of archaeological heritage monuments than in the rest or only some sides of the entire structure. Therefore, their photos are limited to describe their interest for a number of reasons. For instance, the different historical value of different parts of the monuments; the visibility and accessibility for some parts is better than others and finally the parts, which symbolise the monument, are usually much more photographed and published online than any other parts.

\section{MONUMENTS AND DATA SETS}

For our investigations of different image-based 3d reconstruction approaches, we have chosen four important Syrian monuments, which had been destroyed by the so-called Islamic State in 2015: the Umayyad Mosque in Aleppo, the Baalshamin Temple, the Bel Temple and the Arch of Triumph, all three in Palmyra. Apart from their historical importance, these monuments had also been chosen due to their different architectural characteristics and the available imagery database (Table 1 ).

\begin{tabular}{|c|c|c|c|c|}
\hline & \multicolumn{2}{|c|}{ Reconst. $\quad$ Space } & \multicolumn{2}{|c|}{ Avail. Imagery } \\
\hline & Interior & Exterior & Pano. & Touristic \\
\hline O.M.Minaret & No & Yes & Yes & No \\
\hline Bel Temple & Yes & Yes & Yes & Yes \\
\hline Baalshamin & No & Yes & No & Yes \\
\hline A.o.Triumph & No & Yes & Yes & Yes \\
\hline
\end{tabular}

Table 1. The space to reconstruct and the available imagery for every reconstructed monument.

Therefore, different architecture was virtually reconstructed with different image sources types, which was possible using different workflows. The open-domain images were collected online. The professional panoramic photos had been captured by G.Fangi in
2010 before the ongoing Syrian civil war. They have a good coverage and network geometry for every monument. They thus provide an excellent data source and a potential reference framework for the often poorly connected but dense tourist images. The main characteristics of each monument and of the respective data sets are outlined below.

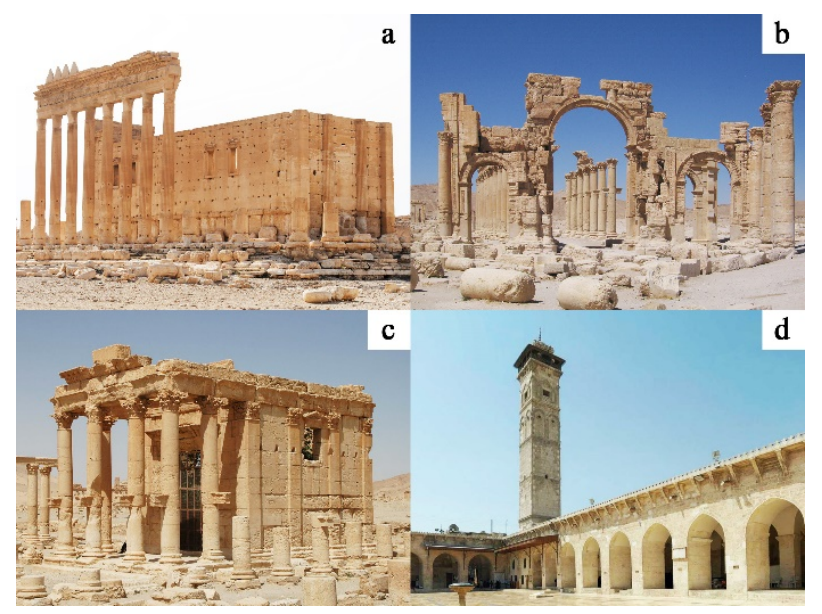

Figure 1. Photos of the three investigated monuments a) Bel temple; b) Arch of Triumph; c) Baalshamin temple; d) the Minaret of the Umayyad mosque in Aleppo.

\subsection{The Minaret of the Umayyad Mosque in Aleppo}

The Great Mosque of Aleppo was built by the Umayyad Caliphs. It is one of the world heritage sites since 1986. Its minaret, dating back to 1090, was built during the Seljuk period. It was an icon of Aleppo city for nearly a thousand years (Figure 1). The minaret was destroyed in 2013. The professional multi-image panoramas were captured in 2010. About 550 photos were captured by a Canon EOS 450D DSLR camera with different focal lengths (50 mm - $200 \mathrm{~mm}$ ). The panoramic stations, distributed around the minaret tower, were used to compose 25 panoramas.

\subsection{Baalshamin Temple in Palmyra}

The Temple of Baalshamin in Palmyra was a first century C.E. sanctuary dedicated to one of the key gods of the city (Figure 1). As with other Palmyrene architecture, the sanctuary of Baalshamin demonstrated hybridity of design incorporating both Near Eastern and Greek-Roman elements. The temple building dated to c. 130 C.E. and represents an addition to a sanctuary that already existed by 17 C.E. For this monument (Becker, 2015). It was destroyed in 2015. We have no professional panoramas but we managed to collect 117 open domain touristic photos with acceptable image dimensions (min 1200 pixels width).

\subsection{Bel Temple in Palmyra}

The Bel Temple was surrounded by a bounded, architectural precinct measuring approximately 205 meters per side. This precinct, surrounded by a portico (a colonnaded entryway), encloses the temple of Bel as well as other cult buildings (Figure 1 ). The inner chamber of the temple stood over 14 meters in height and measured $39.45 \times 13.86$ meters (Becker, 2015). For the image-based reconstruction of the Bel temple, we used two image sources: 180 public domain touristic photos from the web and the panoramic imagery, which was captured using a Canon EOS 450D DSLR camera. The panoramic images cover the interior and the exterior space of the monument with 20 multi- 
image partial panoramas created from 551 individual photos. Thirteen panoramas cover the exterior of the monument and seven panoramas the interior.

\subsection{Arch of Triumph in Palmyra}

The Monumental Arch is one of the symbols of the city (Figure 1). It was built during the reign of Emperor Septimius Severus, which lasted from 193 to $211 \mathrm{AD}$; it linked the main street of the Colonnade and the Temple of Bel. For the virtual reconstruction, we used four panoramas of the Arch composed by a total of 62 images captured with a Canon EOS 450D DSLR camera with fixed focal length and 63 open domain touristic images with good resolution.

\section{RECONSTRUCTIONS STRATEGIES}

The complexity of the monument and the available data sources together with the goal of the model to be reconstructed determine the reconstruction strategies. In our research, we defined three main workflows to $3 \mathrm{~d}$ reconstruct monuments using the available image sources: panoramic image-based modelling, dense multi-view 3d reconstruction and a hybrid approach, combining the these two methods. As photogrammetry software, Agisoft Photoscan was used for the dense multi-view 3d reconstruction investigations. Some of the reasons include its capability to handle a large number of different cameras and the important possibility to introduce geometric constraints into the bundle adjustment.

\subsection{Panoramic image-based modelling}

Panoramic image-based modelling is a modelling process independent from photogrammetric packages, which makes use of equirectangular panoramas and exploits projective geometry rules to produce a metrical model as a survey.

The process is based on the spherical mapping of an image onto $3 \mathrm{~d}$ meshes. Spherical mapping in geometrical terms is a central projection. Which is one of projection possibilities that threedimensional modelling platforms offer. Central projection can be considered as a virtual projector of an image. If we project two different oriented images of the same object, we can find 3d points by forward intersection to create the three-dimensional model. Basing on the points, we can create primitives. Then, the created primitives can be moved and modified to match their projections. Objects therefore, will take the right shape and location in the virtual space of the surveyed elements. While modelling with projected images, the offset or the correspondence between the model and the projected photo is visible interactively (Figure 2).

In many cases, modelling starts from defining key points using forward intersection then using mono-plotting, main planes and features can be defined. Mono plotting means the use of just one projection and some geometrical constraints such as verticality and collinearity. The technique is explained in details in (Wahbeh, 2016) and some interactive video examples are available online such as the modern architecture example on https://youtu.be/akRUJ0G7t2I .

In some cases, the use of open domain photos for a dense multiview 3d reconstruction for producing a good model is not possible. This could be due to a very uneven distribution of the photos or the shape of the monument itself. When the photos are taken far away from the monuments even if captured with high zoom factors, the resulting image network geometries are often very poor with either too small or too large baselines, often resulting in a failing matching and model reconstruction process. a)
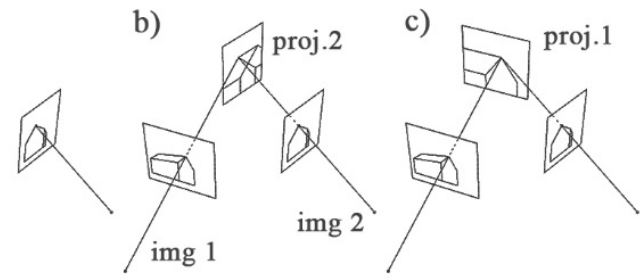

d)

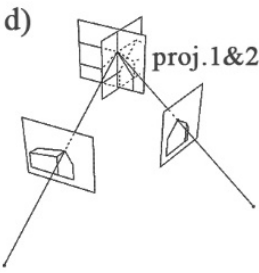

e)
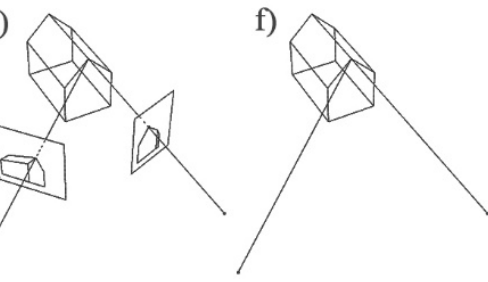

Figure 2. The process of modelling using central projection of oriented images a) projecting two different images b), c) \& d) moving the projections to find the intersection, which means a triangulation e), \& f) completing the model of the architecture.

Even the geometry and the dimensions of the monument has a very important influence on the result.

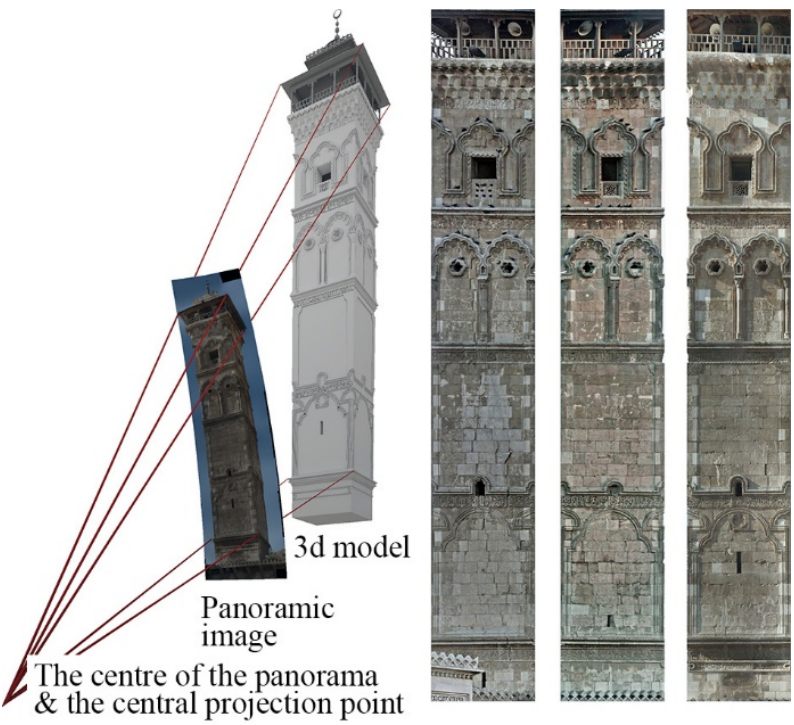

Figure 3. Based on the spatial projection of the oriented panoramas on primitive geometries, it is possible to create the $3 \mathrm{~d}$ model and to extract orthophotos.

In the case of the collapsed minaret of the Umayyad mosque of Aleppo virtually reconstructed by Fangi and Wahbeh (2013) the solution was to use panoramic image-based modelling. In this specific case, the dense multi-view $3 d$ reconstruction technique using the uncalibrated open-domain photos was not successful. This was due to problems related to the configuration of the survey such as the form of the minaret and the relatively large distance to the tourists' photo positions. Using uncalibrated photos for the 3d reconstruction of a thin object like the minaret, which occupies only a small portion of the images, gives inaccurate results expressed by poor estimates for interior and exterior orientations and very noisy point clouds.

Using the professional panoramas of the monument, the virtual reconstruction was possible by means of panoramic image-based modelling. The relative orientation of the panoramas was calculated using the Panoramic Spherical Photogrammetry (PSP) method developed by Fangi (2007). The subsequent modelling process of this method is interactive. The modelling in this case was carried out according to the following steps: 
- $\quad$ Stitching the panoramas from the original image frames;

- Orienting the panoramas using Panoramic Spherical Photogrammetry;

- $\quad$ Setting up a 3d scene with the used panoramas projected according to the orientation parameters;

- $\quad$ Drawing points by forward intersection;

- $\quad$ Creating surfaces and primitives based on retrieved points;

- Projecting the appropriately oriented images onto the surfaces and modifying them according to the projection;

- $\quad$ Finally drawing the small details on the textured surfaces.

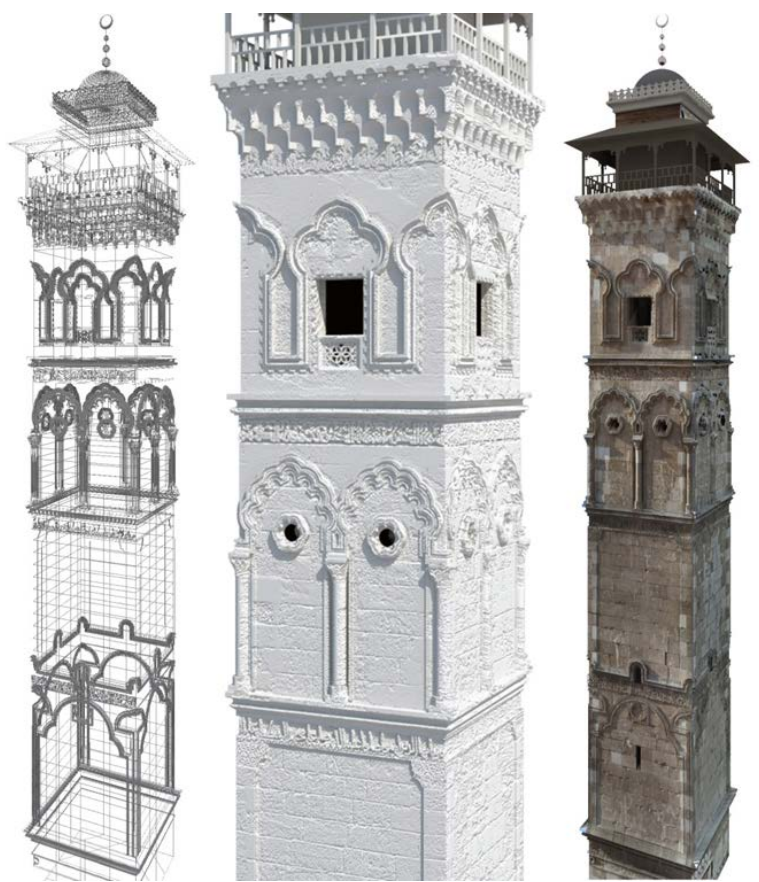

Figure 4. The 3d model of the destroyed minaret of the Umayyad mosque in Aleppo. Performed using panoramic image-based modelling method.

Among the more advanced experiments, being studied is the automation of the process using programming within a $3 d$ modelling platform such as the Grasshopper plugin with Rhinoceros3D. Programming the triangulations aims at connecting directly the collimation of points on the image (input) and the $3 \mathrm{~d}$ modelling (output). The possibility to program the triangulation is convenient to obtain an interactive $3 \mathrm{~d}$ modelling. Therefore, we can get a maximum integration between the survey and the newly constructed model in the same platform. The programmed triangulation approach described in (Wahbeh 2016) so far produces vertices to be used for the $3 \mathrm{~d}$ modelling. It does not yet directly produce a textured mesh as mapping-based modelling method, but it is already much faster and easier.

\subsection{Dense Multi-View 3d Reconstruction}

Dense multi-view 3d reconstruction algorithms, permitting automatic image registration, matching and $3 \mathrm{~d}$ extraction have revolutionised photogrammetry in general and in close-range photogrammetry in particular. New commercial software are able to process huge quantities of images, to calculate the orientation and to produce accurate 3d point clouds, even from uncalibrated photos (Santagati et al., 2013; Koutsoudis et al., 2014). This possibility opened the way for many projects, which use images not originally captured for survey purposes.
If destroyed monuments are to be reconstructed in $3 \mathrm{~d}$ using crowd-sourced images, then they have to be well covered by photos from relatively small distance. The monuments furthermore had to be accessible and photographed from all sides in order to obtain a complete model. Normally, this particular requirement is difficult to fulfil. In case of the $3 \mathrm{~d}$ reconstruction of the building exterior, the monument has to be in the open space and attractive to tourists from all sides. This is applicable to some of the monuments in Palmyra. They are located in the open desert, so they could have been photographed from all the sides.

The investigated two monuments in this section are Baalshamin and Bel temple. They have different characteristics. Baalshamin temple is featured in numerous photos published online but few of them are covering its back façade. In addition, we have no professional panoramas of it. Bel temple is a huge monument with an important interior space to be virtually reconstructed using crowd sourced images and professional panoramas.

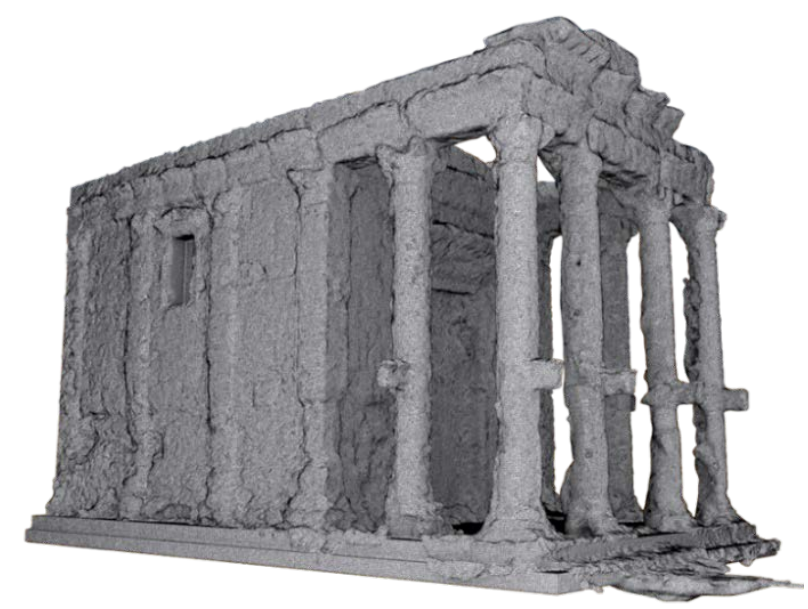

Figure 5. The 3d model mesh of the Baalshamin Temple using dense multi-view $3 \mathrm{~d}$ reconstruction from tourist imagery

The workflow in the case of Baalshamin temple was quite ordinary using these main steps:

- Collection of open-domain images

- Dense multi-view 3d reconstruction, processing the images in Agisoft Photoscan as one chunk and getting the point clouds;

- Creating a mesh from the point clouds;

- Exporting the resulting model for editing;

- $\quad$ Editing the mesh externally to refine it, eliminating visually unrealistic irregularities;

- Importing the model again into the Photoscan environment and texturing the edited mesh. In this case the now reconstructed parts could be textured with images which were useless for the $3 \mathrm{D}$ reconstruction due to the absence of possible triangulations, but that were oriented anyway;

- $\quad$ Exporting the textured mesh to be edited using a virtual sculpting program;

- $\quad$ Refining the texture optimizing the colours balances and contrasts for an optimal representation.

As shown in Figure 5, the dense multi-view reconstruction produced a good model of the exterior of the other three sides. The overall mean re-projection error for all points in all images is 0.72 pixels, which indicates a good quality considering the large number of uncalibrated cameras, used in the process.

In case of the temple interior, it was not possible to produce a single 3d reconstruction and subsequent 3d point cloud of the whole space with a single computation. This was due to the 
distribution and the orientation of the photos owing to the fact that the interesting two chambers with their carved monolithic ceilings were located at the opposite ends of a narrow long rectangle hall. As a result, most of the photos were either oriented towards the chamber at the northern or the southern end. This resulted in an insufficient number of photos for bridging the northern and southern parts of the hall. The solution was to create two image groups and two subsequent point clouds for the interior space. The first group contains the photos oriented towards the northern chamber (Figure 6, top) and the second contains the ones oriented towards the South (Figure 6, middle).

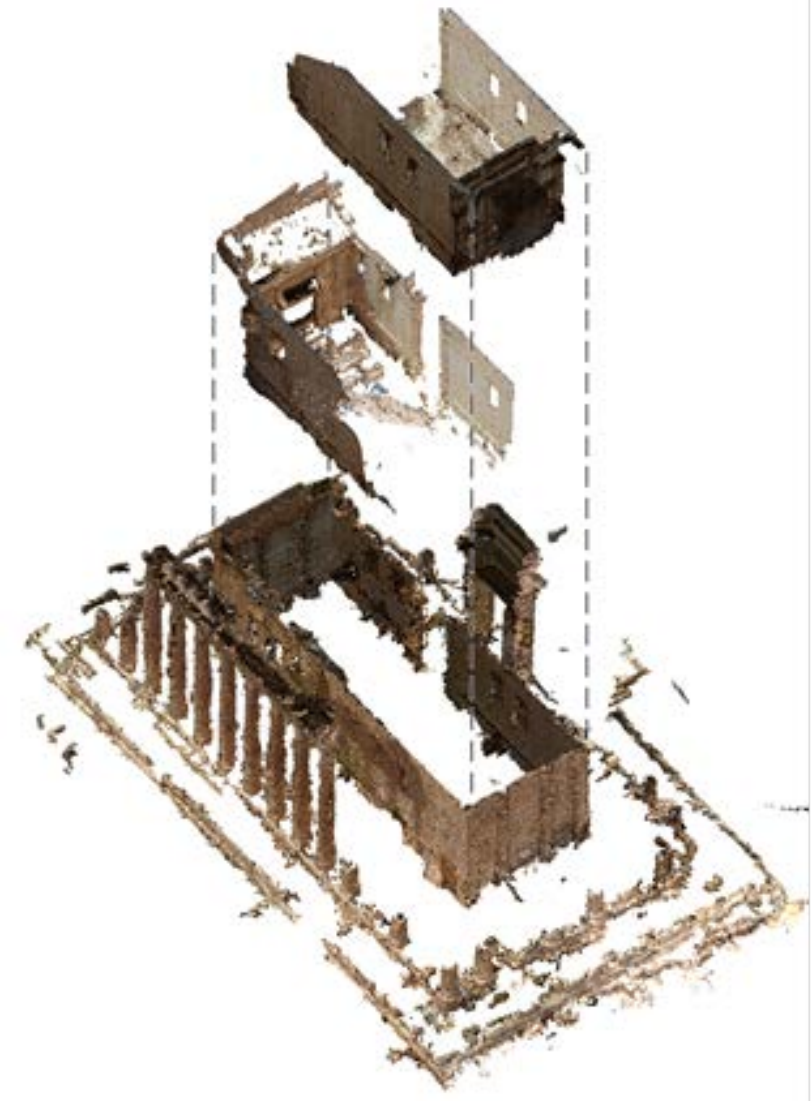

Figure 6. Three groups of images created three point clouds of the temple to be relatively georeferenced.

In all the touristic image groups, we added the professional images, which compose the panoramas and were oriented in a similar direction. Subsequently, the professional and the touristic images were processed together. However, too few images were shared between the two interior image groups to cover the common area.

The process produced the northern interior point cloud with about 8.5 million points, the southern with about 9.5 million points and the exterior one with about 6.5 million points. The overall mean re-projection error for all points in all images is 1.24 pixels. All three point-clouds were referenced to the same local coordinate system using identical natural features as control points.

These main steps summarize the workflow in case of the Bel Temple:

- Collection Professional and open-domain Photos;

- Separating photos into groups based on their distribution and orientation patterns;

- $\quad$ Processing the multi-view 3d reconstruction exploiting geometric constraints in case of the panoramic images (see Wahbeh \& Nebiker, 2016) to produce different point clouds from different chunks;
- The registration of the point clouds using the centre of panoramic images as target points (Wahbeh, 2016);

- Generating the mesh form the combination of point clouds

- $\quad$ Post processing the mesh in an external mesh editor to optimize it;

- Importing the mesh again into the photogrammetry software scene and texture it;

- $\quad$ Post processing the textured mesh optimizing the colours and the contrast for the final visualization.

The final product was a completely textured model - apart from some portions of the pavement (Figure 7). The final model also has a good metric quality with a relative accuracy in the order of $2-3 \mathrm{~cm}$ and an absolute accuracy in the order of $10-15 \mathrm{~cm}$, which is equivalent to better than $0.3 \%$ of the Temple dimensions (Wahbeh \& Nebiker, 2016).
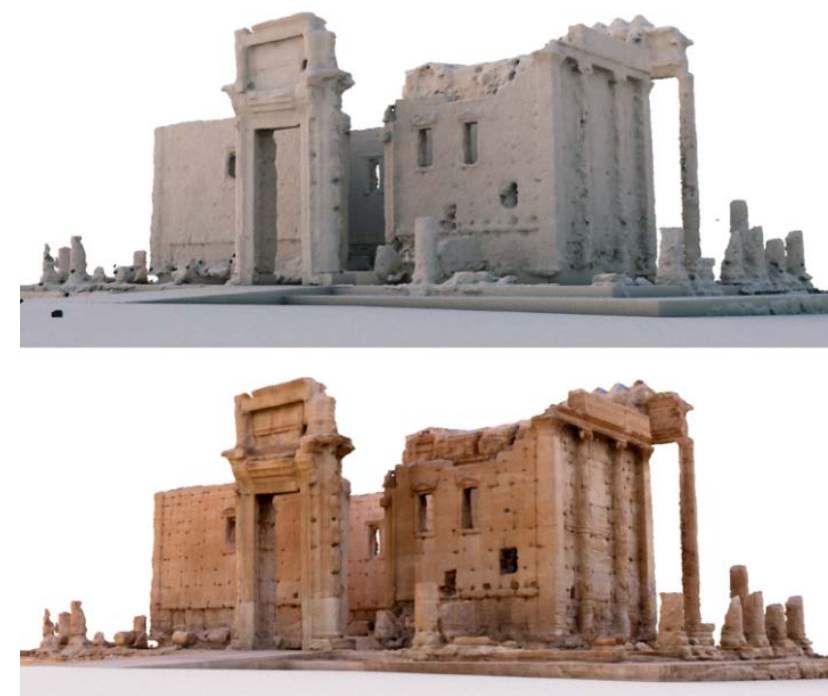

Figure 7. The final model of Bel Temple (untextured: top, textured: bottom)

\subsection{Hybrid virtual reconstruction process}

In a typical photogrammetric survey for a complete 3d model of an object, photos from a large number of viewpoints are required. Today, ideally a combination of UAV- and ground-based images possibly in combination with single or multi-image panoramas are used. Thus, most often the combination of more than one image source and survey technology is useful to complete or improve the results.

Taking the Arch of Triumph as example: Starting with the dense multi-view 3d reconstruction based on some professional photos and many open-domain ones, the produced mesh model contained many missing parts as mentioned in the previous chapter. After reconstructing the initial mesh, and before texturing it, the model had to be completed using the second mentioned approach (Figures 8 \& 9). It worked quite well overall because the geometries are clear and regular in this case. It is more laborious when there are irregular parts, which need to be simplified. Then the surfaces could be reconstructed with a lower level of detail but they could deliver a better virtual view of the original state of the monument especially in combination with textures. 


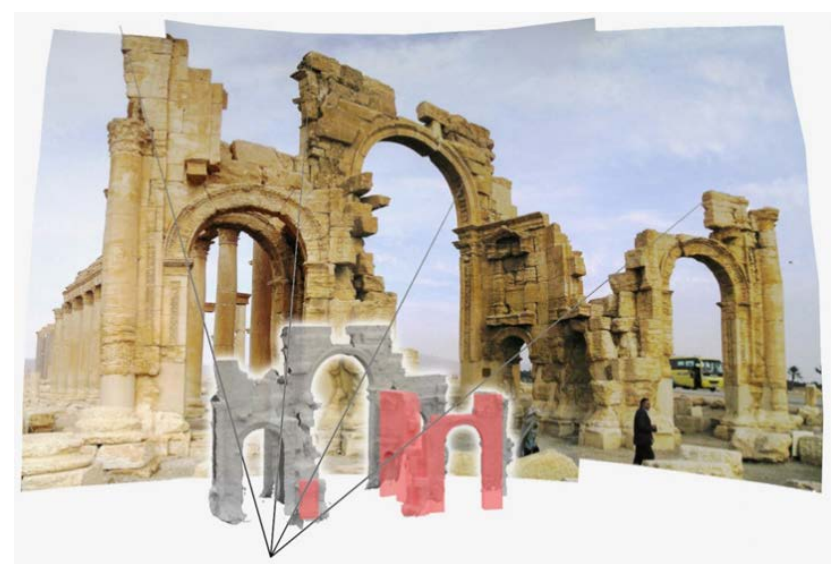

Figure 8. The destroyed Arch of Triumph in Palmyra, a projection of a panorama and the reconstructed model. Shown in grey is the part from dense multi-view $3 \mathrm{~d}$ reconstruction and in red is the part from panoramic image-based modelling.

In the specific case of the Arch of Triumph, we combined the above-mentioned two techniques dense multi-view 3d reconstruction and Panoramic image-based 3d reconstruction using the following workflow:

- The collection of professional and open-domain images and classification into two groups basing on the orientation and distribution patterns;

- Dense multi-view 3d reconstruction, processing the chunks and getting the point clouds;

- From the process we could retrieve the positions of the professional panoramic images;

- The importation of the mesh in 3d modelling software and projecting the panoramas using central projections from the retrieved positions. The correct orientation could be obtained graphically according to the imported mesh;

- Completing the $3 \mathrm{~d}$ model according to the projections to obtain two types of meshes: a dense mesh and low poly one;

- Creating one closed solid mesh resampling all the pieces together. A function that is possible in some $3 \mathrm{~d}$ modelling tools such as "Mesh Mixer";

- Importing the model again into the photogrammetry program and texturing it;

- Post processing the texture optimizing colour balance and contrast to finish the model for the appropriate representation.

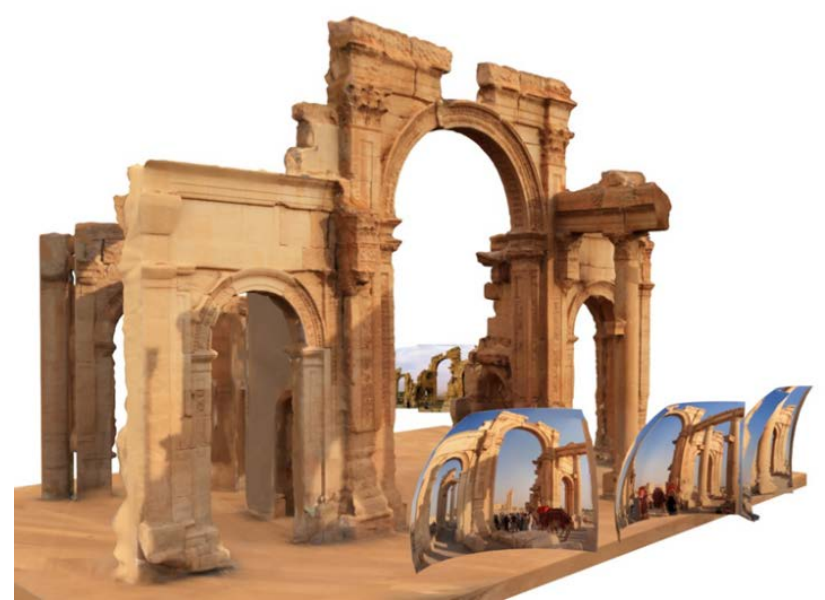

Figure 9. The final model of the Arch of Triumph and the used panoramas.

\section{CONCLUSIONS AND OUTLOOK}

Workflows need to be adapted to the source images and the reconstructed object if we want to complete as much as possible a model even using one specific technique such as dense multiview $3 \mathrm{~d}$ reconstruction. The innovation in our case is in the use of $3 \mathrm{~d}$ modelling as a photogrammetric tool. The application of photogrammetric fundamentals in the $3 \mathrm{~d}$ modelling process turns the 3d modelling software into a photogrammetric tool. Furthermore, it becomes a platform to integrate the two different modelling products to complete a survey model artefact. The multi-pass workflow combines automatic and non-automatic processes to obtain a model with optimal accuracy and completeness for the documentation, representation and finally the conservation of the memory of the lost heritage monuments.

Among our ongoing work is the inclusion of quality parameters in the $3 \mathrm{~d}$ reconstruction process and the interactive visualisation of the models. This takes into account that different parts of (destroyed) monuments were covered by different numbers, configurations, densities, and types of imagery. As a consequence, the resulting image-based reconstructions will lead to models with varying levels of detail, accuracy and even completeness. With our ongoing research we aim at propagating this information through the modelling process and giving the users of the model direct access to this quality information, e.g. by means of additional texture layers.

\section{ACKNOWLEDGEMENTS}

These virtual reconstructions had not been possible without the rich documentation of many Syrian monuments by Gabriele Fangi. His ongoing passion in photogrammetrically documenting heritage monuments around the world is now bearing fruits, especially in this area where important cultural heritage monuments are in real danger.

\section{REFERENCES}

Agarwal, S., Furukawa, Y., Snavely, N., Simon, I., Curless, B., Seitz, S.M., Szeliski, R., 2011. Building rome in a day. Commun. ACM 54, 105-112.

Alsadik, B., Gerke, M., Vosselman, G., Daham, A., \& Jasim, L. (2014). Minimal camera networks for 3D image based modeling of cultural heritage objects. Sensors, 14 (Oct 2015), 5785-804. https://doi.org/10.3390/s140405785

Becker J. A., 2015 Essay on Bel and Baalshamin temples in Palmyra www.khanacademy.org

Fangi, G., 2007. The Multi-image spherical Panoramas as a tool for Architectural Survey, in: 21th CIPA Symposium. pp. 256-1840.

Fangi, G., Wahbeh, W., 2013. The Destroyed Minaret of the Umayyad Mosque of Aleppo, the Survey of the Original State. European Scientific Journal ESJ 9(10).

Gruen, A., Remondino, F., Zhang, L., 2002. Reconstruction of the Great Buddha of Bamiyan, Afghanistan. ISPRS Arch. Photogramm. Remote Sens.

Kádár, B., Gede, M., 2013. Where Do Tourists Go? Visualizing and Analyzing the Spatial Distribution of Geotagged 
Photography. Cartographica 48, 78-88. doi:10.3138/carto.48.2.1839

Kersten, T.P., Lindstaedt, M., 2012. Automatic 3D Object Reconstruction from Multiple Images for Architectural, Cultural Heritage and Archaeological Applications Using Open-Source Software and Web Services. Photogramm. - Fernerkundung Geoinf. 2012, 727-740. doi:10.1127/1432-8364/2012/0152

Koutsoudis, A., Vidmar, B., Ioannakis, G., Arnaoutoglou, F., Pavlidis, G., Chamzas, C., 2014. Multi-image 3D reconstruction data evaluation. J. Cult. Herit. 15, 73-79. doi:10.1016/j.culher.2012.12.003

Santagati, C., Inzerillo, L., Paola, F. Di, 2013. Image-based modeling techniques for architectural heritage 3d digitalization: limits and potentialities. ISPRS Arch. Photogramm. Remote Sens. XL, 2-6. doi:10.5194/isprsarchives-XL-5-W2-555-2013

Wahbeh, W., 2016. From Spherical Photogrammetry to 3D Modeling. Handb. Res. Vis. Comput. Emerg. Geom. Des. Tools 1, 96-115.

Wahbeh, W., Nebiker, S., Fangi, G., 2016. Combining Public Domain and Professional Panoramic Imagery for The Accurate and Dense 3d Reconstruction of The Destroyed Bel Temple in Palmyra. ISPRS Ann. Photogramm. Remote Sens. Spat. Inf. Sci. III-5, 81-88. doi:10.5194/isprs-annals-III-5-81-2016 